\title{
Two-stage Stochastic Model using Benders' Decomposition for Large-scale Energy Resources Management in Smart grids
}

João Soares, Member, IEEE, Bruno Canizes, M. Ali Fotouhi Ghazvini, Zita Vale, Senior Member, IEEE GECAD - Research Group on Intelligent Engineering and Computing for Advanced Innovation and Development Polytechnic of Porto, 4200-072, Porto, Portugal \{joaps,brmrc,mafgh,zav\}@isep.ipp.pt

G. K. Venayagamoorthy, Senior Member, IEEE

Real-Time Power and Intelligent Systems Lab. Clemson University, Clemson, SC 29634, USA and School of Engineering, University of KwaZulu-Natal, Durban, South Africa gkumar@ieee.org braging a smart grid. The idea is to address the chall brought by the variability of demand, renewable energy, electric vehicles, and market price variations while pursuing cost minimization. Benders' decomposition approach is implemented to improve the tractability of the original model and its' computational burden. A realistic case study is presented using a real distribution network in Portugal with high penetration of renewable energy and electric vehicles. The results show the effectiveness and efficiency of the proposed approach when compared with a deterministic formulation and suggest that demand response and storage systems can mitigate the uncertainty.

Index Terms--Benders decomposition, Energy management, Large-scale systems, Optimization methods, Power generation scheduling, Stochastic systems, Uncertainty

\section{NOMENCLATURE}

\section{Indices}

$b$

e

$i$

$l$

$s$

$s p$

$t$

$v$

Parameters

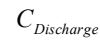

$C_{D G}$

$C_{\text {LoadDR }}$

$C_{Q C P}$

$C_{\mathrm{SSD}}$

$C_{\text {Supplier }}$

$E_{\text {BatCap }}$

$E_{\text {Min Charge }}$

$M P$

$N_{i}$
Electrical buses

Energy storage systems (ESSs)

Distributed generation units (DG)

Loads

Scenarios

External suppliers

Timeslot

Electric vehicles (EVs)

Discharging cost of ESSs/EVs [m.u./kWh]

Generation cost of DG units [m.u./kWh]

Load reduction cost [m.u./kWh]

Curtailment cost of DG units [m.u./kWh]

Non-supplied demand cost [m.u./kWh]

External suppliers cost [m.u./kWh]

Capacity of ESSs/EVs batteries [kWh]

Minimum energy stored in ESSs/EVs [kWh]

Market price [m.u./kWh]

Number of DG units
$N_{e}$

$N_{l}$

$N_{s}$

$N_{v}$

$P_{\text {Chargelim }}$

\section{Number of ESSs}

Number of loads

Number of external electricity suppliers

Number of EVs

Maximum charge rate of ESSs/EVs [kW]

Forecasted generation of non-dispatchable DG [kW]

$P_{D G M i n L i m i t}$

$P_{\text {DGMaxLimit }}$

Min. active power of dispatchable DG [kW]

Max.active power of dispatchable DG [kW]

Maximum discharge rate of ESSs/EVs [kW]

Maximum power reduction of loads [kW]

Maximum offer allowed in market [kW]

Minimum offer allowed in market $[\mathrm{kW}]$

Maximum buy allowed in market [kW]

Minimum buy allowed in market [kW]

Minimum active power of suppliers [kW]

Maximum active power of suppliers [kW]

Number of time periods

Number of scenarios

Lagranges from slave in $m$-1 iteration

Duration of period $t(1=$ hour $)$

Occurrence probability of scenarios

Charging efficiency of ESSs/EVs

Discharging efficiency of ESSs/EVs

Variables

$P_{B u y}$

$p_{\text {Charge }}$

$p_{\text {Discharge }}$

$p_{D G}$

$p_{\text {LoaddR }}$

$p_{G C P}$

$p_{N S D}$

$P_{\text {Sell }}$

$E_{\text {Stored }}$

$p_{\text {Supplier }}$

$x_{D G}$

$x_{E S S E V}$
Active power bid in market [kW]

Active power charging of ESSs/EVs [kW]

Active power discharge of ESSs/EVs [kW]

Active power of dispatchable DG [kW]

Active power reduction of loads [kW]

Generation curtailment power of DG [kW]

Active power of NSD of load [kW]

Active power offer in market [kW]

Energy stored in ESS/EVs [kWh]

Active power of external suppliers [kW]

Binary variable of state of DG units

Binary variable representing discharging 


$\begin{array}{ll}y_{E S S / E V} & \begin{array}{l}\text { state of ESSs/EVs } \\ \text { Binary variable representing charging state } \\ \text { of ESSs/EVs } \\ \text { Binary variable that represents the choice of } \\ x_{\text {Market }}\end{array} \\ x_{\text {Supplier }} & \begin{array}{l}\text { markets } \\ Z A\end{array} \\ Z F & \text { Binary variable of choosing suppliers } \\ \text { Sets } & \text { Relaxation variable in Benders slave (bus) } \\ \Omega_{D G}^{d} & \text { Dispatchable DG units } \\ \Omega_{D G}^{n d} & \text { Non-dispatchable DG units } \\ \Omega_{D G}^{b} & \text { DG units connected to bus } b \\ \Omega_{E}^{b} & \text { ESSs connected to bus } b \\ \Omega_{H}^{b} & \text { Lines connected to bus } b \\ \Omega_{L}^{b} & \text { Loads connected to bus } b \\ \Omega_{S P}^{b} & \text { External suppliers in bus } b \\ \Omega_{V}^{b} & \text { EVs connected to bus } b\end{array}$

\section{BACKGROUND}

Renewable energy sources present a high level of variability concerning energy generation. This unpredictability should be managed efficiently by smart grid (SG) technologies. Conceptually, SG is meant to be highly flexible in order to accommodate high penetration of renewable energy while being able to attenuate uncertainty.

Transactive energy systems can contribute to delivering the flexibility required by the smart grid, e.g. controllable loads, including electric vehicles (EVs) under interoperable architectures [1], [2]. This flexibility can be provided through energy aggregators, which are meaningful for small producers under market-oriented environment [3]. To allow efficient and cost effective operation, energy aggregators need suitable energy resources management (ERM) tools to deal with the increasing number of resources and its underlying uncertainty, e.g. EVs and renewables [4], [5]. The day-ahead energy scheduling is an important part of an ERM system to obtain the expected operation cost (or profit) while providing adequate decisions one day in advance. However, the energy scheduling is quite challenging due to the inherent uncertainties and the high number of resources.

Adopting advanced energy management models that consider uncertainty factors are critical for successful implementation of SGs. The United States Department of Energy (DOE) has identified predictive models to deal with stochastic behavior and uncertainty as a top R\&D priority [6].

The day-ahead problem tackled by this paper is a combinatorial problem of large-scale nature when many distributed energy resources (DERs) are considered. Due to nonlinearity features of the problem, it is usually classified as mixed integer nonlinear programming (MINLP). MINLP techniques require significant computer resources. The computation time needed for solving these types of problems is not compatible with the time limitations of short-term energy scheduling [7].
To overcome the computational burden issue, some approaches have been proposed in previous research. The work developed in [8] adopts Benders' decomposition approach to solve a multi-objective model in day-ahead context. The authors were able to reduce the complexity of the original MINLP scheduling problem compared to a previous formulation proposed in [7]. However, it was found later in [9], that the slave problem formulated as an hourly distribution power flow in [8] leads to sub-optimal solutions, due to temporal dependencies in distributed energy resources. Therefore, the work in [9] proposes a multi-period model to obtain better results. Furthermore, the work in [8] seems limited in the sense that it does not consider demand response (DR), renewable generation such as wind or PV, and energy storage systems (ESS), which are increasingly important in SGs. Although the proposed works have contributed to reducing the original problem complexity, uncertainty factors have not been considered in the mentioned works [7]-[9] and many others presented in the literature [10]-[15].

Energy scheduling models that incorporate stochasticity have been proposed already. In [16], a dispatch scheduling approach is proposed for a wind farm using ESSs. The results indicate that the ESS can be used to perform a joint production schedule and address the forecasting errors during the real-time operation. A study using stochastic energy management with compressed air storage integrated with renewable generation has demonstrated to be effective in [17]. The works proposed in [18], [19] focuses on aggregator's market strategies and the risks associated with their portfolio optimization problems. Furthermore, it is suggested that the model may be decomposable and subject of future research [19]. In [20], a stochastic model is proposed to address the ERM in hybrid $\mathrm{AC} / \mathrm{DC}$ microgrids considering DERs and uncertainty in EV demand, renewable generation, and electricity price. However, DR is not considered in the work above and it only considers a small power system (38bus) with 8 DG units. The model is adequate for small hybrid $\mathrm{AC} / \mathrm{DC}$ grids whereas the proposed model in this paper is targeted to deal with larger grids. In [21], authors present a stochastic day-ahead scheduling to address carbon emission, generation fuel costs and uncertainties in microgrid operation. The work does not incorporate network constraints and the experiments are based on a small 3 generator system. The work presented in [22] tackles the ERM problem of a renewable-based virtual power plant. These models consider the uncertainty in electricity prices and renewables, but the consideration of resources such as DR, EVs, and V2G capacity have been overlooked. The use of energy resources (e.g. ESS) can mitigate system uncertainties as demonstrated in [4], [16], [17], [22], [23]. Nevertheless, these works do not consider EVs and related uncertainties, which are expected to grow considerably in next decade. Other works consider the EV uncertainty [20], [24] but do not incorporate grid constraints. When the grid is included in the stochastic models, it is either decoupled or only suited for a small grid system with few scheduling units. In this paper, the proposed model attempts to overcome this issue by using a stochastic Benders' decomposition, which allows to include network 
constraints, a high number of DERs, EVs and several sources of uncertainty in the same model without requiring external validation, while still allowing scalability and good results. The present research paper takes into account the lessons learned from [9], where Benders' decomposition is proposed to address energy resources scheduling considering several kinds of DERs and network constraints. In the current research, a two-stage stochastic model research is proposed to incorporate the ability to handle uncertainty factors, which was not tackled in [9]. The Benders' decomposition scheme is applied to the two-stage stochastic model to reduce the computational burden of the large-scale problem. In addition, several modifications to the original optimization model have been undertaken to allow the method to handle several representative scenarios efficiently. A realistic case study using a real 180-bus grid from Portugal with high penetration of DERs is used to demonstrate the application of the method.

This paper is structured as follows: Section II describes the data uncertainty involved in this problem and how the scenarios are generated; Section III describes the problem formulation; Section IV presents the case study used in this work, while Section $\mathrm{V}$ presents the results and respective discussion. Finally, the last section presents the main conclusions of the paper.

\section{MODELLING SYSTEM UNCERTAINTIES}

The aggregator in this model faces several sources of uncertainty during the operation of active distribution networks, namely the forecast errors of EV fleet characteristics, hourly load demands and the generation profile of the renewable sources. They are considered as potential uncertainties [25]. The uncertainties related with these inputs are taken into account in the model and the scheduling problem is developed as a stochastic scenariobased optimization model. The uncertainties associated with the EV fleet characteristics is caused by the random driving pattern of the EV drivers and their uncertain behavior [25].

In this form of problems, where a set of scenarios needs to be handled, the main issue is to generate a set of realizations for the random variable. These scenarios should adequately represent the probabilistic characteristics of the data [26]. In this stochastic scheduling model, the initial set of scenarios is a large data set generated by the Monte Carlo Simulation (MCS) technique for representing power system uncertainties which the decision-maker faces while solving the problem. The MCS parameters are the probability distribution functions of the forecast errors [27].

In order to include the forecast error, an additional term which can be positive or negative is added to the forecasted profile ( $x^{\text {forecasted }}$ )

$$
x^{s}(t)=x^{\text {forecasted }}(t)+x^{\text {error }, s}(t), \quad \forall t, \forall s .
$$

The error term $\left(x^{\text {error,s }}\right)$ is a zero-mean noise with standard deviation $\sigma$ [26], [28]. Scenarios are represented with $x^{s}$ The uncertainties of the forecast errors are modeled with the probability distribution functions, which are obtained from the historical data [26]. In this model, the forecast errors for the uncertain inputs are all represented by normal distribution functions.

The scenario tree concept can clearly illustrate how the discrete outcome for each stochastic input can be combined to construct the larger set of scenarios. A scenario tree consists nodes that represent the states of the random variable at particular time points, branches to show different realizations of the variable and the root which shows the beginning point where the first stage decisions are made [26]. Fig. 1 shows the scenario tree model for the proposed scenario-based stochastic programming model [26]. $x_{n}^{s}$ refers to the $n^{\text {th }}$ random variable. Variables can have different natures. For instance, $x_{1}^{s}$ may represent load demand and $x_{2}^{s}$ can denote market prices. The number of the nodes at the second stage is equal to the total number of scenarios. The occurrence probability of each scenario is equal to the product of the branches' probabilities [26].

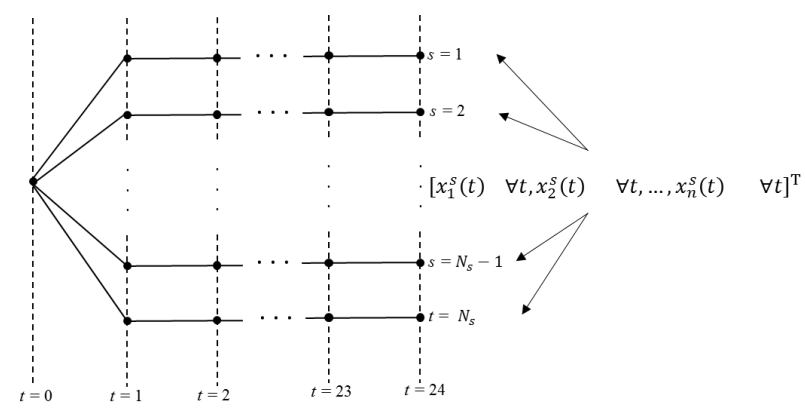

Fig. 1. Scenario tree representation

Including all the generated scenarios in the optimization problem results in a large-scale optimization problem [26]. Generally, there should be a tradeoff between model accuracy and computation speed [25], [29]. In order to handle the computational tractability of the problem, the standard scenario reduction techniques developed in [30] is used. These scenario reduction algorithms exclude the scenarios with low probabilities and combines those that are close to each other in terms of statistic metrics [30]. They determine a scenario subset of the prescribed cardinality and probability which is closest to the initial distribution in terms of a probability metric [27]. The main purpose of scenario reduction is to reduce the size of the problem. The number of variables and equations are reduced after applying these algorithms. Consequently, the solutions can be found more efficiently, without losing the main statistical characteristics of the initial dataset [31]. However, the potential cost of applying these approaches is introducing imprecision in the final solution [29]. The reduction algorithms proposed in [30] consists of algorithms with different computational performance and accuracy, namely fast backward method, fast backward/forward method and fast backward/backward method. The selection of the algorithms depends on the problem size and the expected solution accuracy [27], [30]. For instance, the best computational performance with the worst accuracy can be provided by the fast backward method for large scenario tress. Furthermore, the forward method provides best accuracy and highest computational time. Thus, 
it is usually used where the size of reduced subset is small [27].

\section{PROBlem Formulation}

In this section, the two-stage stochastic model is formulated for the envisaged energy scheduling problem in day-ahead. The main objective of the proposed model is to obtain the optimal decision, namely the day-ahead market transactions and the controllable generation scheduling (first stage). The decision is made taking into account possible deviations in the operation, like wind and solar power and EVs (second stage). The goal is to obtain the lowest operation cost for the energy aggregator, namely the expected operation cost, represented by $E$. The stochastic model depends on the scenario generation approach discussed in the previous section. The scenarios represent the underlying uncertainty of the problem (i.e. the possible deviations from the forecasted values). It means that the wind and solar generation or the load demand varies from one scenario to another. The firststage decisions do not change across the scenarios in the second stage. In other words, the decisions to be made one day in advance remain unchanged.

The two-stage stochastic model is decomposed in two smaller problems, namely the master problem and the slave problem using the Benders' decomposition theoretical approach. The aim is to make the model computationally tractable, as discussed in subsection B.

\section{A. Two-stage stochastic model}

The outputs of this optimization model are the decision variables regarding the purchases from the external suppliers, optimal bids to the wholesale market and the dispatch of the controllable DG units. The total expected operation cost for the day-ahead operation, $D+1$, is represented by (2), corresponding to the first-stage operation costs $\left(O C^{1}\right)$ and second-stage operation costs $\left(O C^{2}\right)$ and market transactions $(M T)$. Theoretical background on stochastic programming models can be found in [32].

Minimize $E\left(O C_{\text {Total }}^{D+1}\right)=O C^{1}+E\left(O C^{2}+M T\right)$

The expected operation cost in the first stage, $O C^{1}$, is represented by (2), which includes the cost of controllable DG units and external suppliers' electricity acquisition:

$$
O C^{1}=\sum_{t=1}^{T}\left[\left(\begin{array}{l}
\sum_{I \in \Omega_{D G}^{d}} p_{D G(i, t)} \cdot C_{D G(i, t)}+ \\
\sum_{s p=1}^{N_{s p}} p_{\text {Supplier }(s p, t)} \cdot C_{\text {Supplier }(s p, t)}
\end{array}\right) \cdot \Delta t\right]
$$

The expected operation cost in the second stage, $O C^{2}$, is represented by (3), which includes the cost of nondispatchable DG units, demand response, ESS/EVs discharge, non-supplied demand (NSD) and generation curtailment power (GCP).

$$
\begin{aligned}
& E\left(O C^{2}\right)=
\end{aligned}
$$

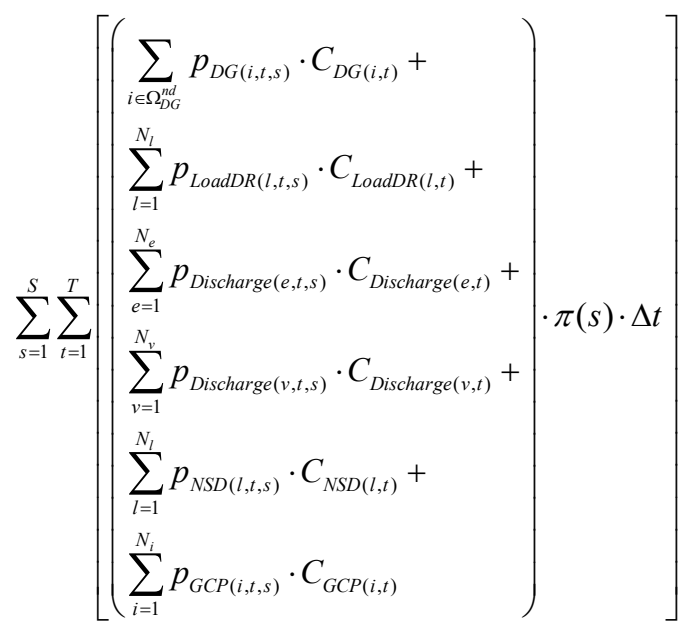

The expected cost of the market transactions are represented by (5), namely the difference between cost of market bids and offers' revenue.

$E(M T)=$

$\sum_{s=1}^{S} \sum_{t=1}^{T}\left[\left(p_{\text {Buy }(t)} \cdot M P_{(t, s)}-p_{\text {Sell }(t)} \cdot M P_{(t, s)}\right) \cdot \pi(s) \cdot \Delta t\right]$

The "here and now" variables which are known as first stage variable are $p_{B u y}, p_{S e l l}, p_{D G}$, and $p_{\text {Supplier }}$. They are determined before the uncertainty is revealed.

\section{1) Network grid constraints}

The DC power flow constraints have been incorporated in the optimization model (6). The usage of a DC model is justified because in many countries, like in Portugal, the distribution networks have voltage regulators and capacitors banks carefully positioned along the grid in order to keep the voltage and reactive power between the desire limits. Thus, in this case the complete model would only make the method more complex and computationally intractable. Usually, the voltage stability is placed at the HV/MV substation level. However, in Portuguese case the MV/LV transformers also have voltage regulators. When $b=1$ in (5), the term $\left(p_{\text {Sell }(t)}-p_{B u y(t)}\right)$ is subtracted to the left part of the equation. It is assumed that the upstream grid is connected to the distribution network at $b=1$.

$$
\begin{aligned}
& \sum_{i \in \Omega_{D G}^{b}}\left(p_{D G(i, t)}-p_{G C P(i, t)}\right)+\sum_{s p \in \Omega_{S P}^{b}} p_{\text {Supplier }(s p, t)}+ \\
& \sum_{l \in \Omega_{L}^{b}}\left(p_{N S D(l, t, s)}+p_{\text {Load } D R(l, t, s)}-p_{\text {Load }(l, t, s)}\right)+ \\
& \sum_{e \in \Omega_{E}^{b}}\left(p_{\text {Discharge }(e, t, s)}-p_{\text {Charge }(e, t, s)}\right)+ \\
& \sum_{v \in \Omega_{V}^{b}}\left(p_{\text {Discharge }(v, t, s)}-p_{\text {Charge }(v, t, s)}\right)- \\
& \sum_{h \in \Omega_{H}^{b}} p_{\text {Line }(h, t, s)}=0 \quad \forall t ; \forall s ; b \neq 1
\end{aligned}
$$


The maximum admissible line flow is expressed by (7) and (8).

$$
\begin{aligned}
& p_{\text {Line }(h, t, s)} \leq P_{\text {LineMaxRate }(h, k)} \quad \forall t ; \forall s \\
& p_{\text {Line }(h, t, s)} \geq-P_{\text {LineMaxRate }(h, k)} \quad \forall t ; \forall s
\end{aligned}
$$

\section{2) DG units and external supplier}

A binary variable is used to represent the commitment status of dispatchable DG units. A value of 1 means that the unit is connected. The non-dispatchable DG active power is fixed according to (11). Maximum and minimum limits for active power in each period $t$ are formulated as:

$$
\begin{aligned}
& p_{D G(i, t)} \geq x_{D G(i, t)} \cdot P_{D G M i n L i m i t(i, t)} \quad \forall t, \forall i \in \Omega_{D G}^{d} \\
& p_{D G(i, t)} \leq x_{D G(i, t)} \cdot P_{D G M a x L i m i t(i, t)} \quad \forall t, \forall i \in \Omega_{D G}^{d} \\
& p_{D G(i, t, s)}=P_{D G S c e n a r i o(i, t, s)} \quad \forall t, \forall i \in \Omega_{D G}^{n d}, \forall s
\end{aligned}
$$

The upstream supplier limits in each period $t$ regarding active power can be formulated as:

$$
\begin{array}{ll}
p_{\text {Supplier }(s p, t)} \geq x_{\text {Supplier }(s p, t)} \cdot P_{\text {SMinLimit }(s p, t)} & \forall t, \forall s p \\
p_{\text {Supplier }(s p, t)} \leq x_{\text {Supplier }(s p, t)} \cdot P_{\text {SMaxLimit }(s p, t)} & \forall t, \forall s p
\end{array}
$$

\section{3) Energy storage systems constraints}

The constraints for the ESS (batteries) are described below. The ESS charge and discharge cannot be simultaneous. Therefore, two binary variables guarantee this condition for each ESS:

$$
x_{E S S(e, t, s)}+y_{E S S(e, t, s)} \leq 1 \quad \forall t, \forall e, \forall s
$$

The battery balance for each ESS $e$ is formulated as:

$$
\begin{aligned}
& E_{\text {Stored }(e, t, z)}=E_{\text {Stored }(e, t-1, s)}+ \\
& \eta_{c(e)} \cdot p_{\text {Charge }(e, t, s)} \cdot \Delta t-\frac{1}{\eta_{d(e)}} \cdot p_{\text {Discharge }(e, t, s)} \cdot \Delta t \quad \forall t, \forall e, \forall s
\end{aligned}
$$

The maximum discharge limit for each ESS is represented by:

$$
p_{\text {Discharge }(e, t, s)} \leq P_{\text {DischargeLimit }(e, t)} \cdot x_{E S S(e, t, s)} \quad \forall t, \forall e, \forall s
$$
by

The maximum charge limit for each ESS is represented

$$
p_{\text {Charge }(e, t, s)} \leq P_{\text {Chargelimit }(e, t)} \cdot y_{E S S(e, t, s)} \quad \forall t, \forall e, \forall s
$$

The maximum battery capacity limit for each ESS is represented by:

$$
E_{\text {Stored }(e, t, s)} \leq E_{\text {BatCap }(e)} \quad \forall t, \forall e, \forall s
$$

Minimum stored energy to be guaranteed at the end of period $t$ is represented by:

$$
E_{\text {Stored }(e, t, s)} \geq E_{\text {MinCharge }(e, t)} \forall t, \forall e, \forall s
$$

\section{4) Electric vehicles constraints}

The EVs are treated as virtual batteries in the proposed model. A virtual battery may represent a parking lot or a set of EVs located in a given network point, which can be estimated in advance. The considered technical constraints are very similar to the formulation provided to the ESSs. However, they have been formally separated to better identify the related variables and because some parameters present source of uncertainty, due to EVs randomness.

The virtual battery charge and discharge limit varies for each scenario $s$, as well as its capacity. This depends on the number of EVs in each bus and the time slot. The maximum discharge limit for each battery $v$ is represented by:

$$
p_{\text {Discharge }(v, t, s)} \leq P_{\text {DischargeLimit }(v, t, s)} \cdot x_{E V(v, t, s)} \quad \forall t, \forall v, \forall s
$$

The maximum charge limit for each virtual battery $v$ is represented by:

$$
p_{\text {Charge }(v, t, s)} \leq P_{\text {ChargeLimit }(v, t, s)} \cdot y_{E V(v, t, s)} \quad \forall t, \forall v, \forall s
$$

The maximum battery capacity limit for each virtual battery $v$ is represented by:

$$
E_{\text {Stored }(v, t, s)} \leq E_{\text {BatCap }(v, s)} \quad \forall t, \forall v, \forall s
$$

The minimum stored energy to be guaranteed at the end of period $t$ is stochastic and is represented by:

$E_{\text {Stored }(v, t, s)} \geq E_{\text {MinCharge }(v, t, s)} \forall t, \forall e, \forall s$

\section{5) Demand response constraints}

Equation (25) formulates a DR load model, namely the direct load control. The maximum amount that each load $l$ can be reduced in each period $t$ in scenario $s$ is formulated as:

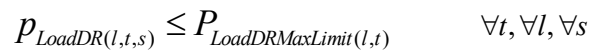

\section{6) Electricity market constraints}

The stochastic energy scheduling model is compatible with the possibility to make bids (buy or sell) to a wholesale market [33]. The energy aggregator may limit the bids within certain bounds. In certain electricity markets, there is a minimum required amount to access.

The market offers are constrained by (28) and (29), namely maximum and minimum offer:

$$
\begin{array}{ll}
p_{\text {Sell }(t)} \leq P_{\text {MarketOfferMax }(t)} \cdot x_{\text {MarketSell }(t)} & \forall t \\
p_{\text {Sell }(t)} \geq P_{\text {MarketOfferMin }(t)} \cdot x_{\text {MarketSell }(t)} & \forall t
\end{array}
$$

The market bids (buy) are constrained by (26) and (27), namely by maximum and minimum amount:

$$
\begin{array}{ll}
p_{\text {Buy }(t)} \leq P_{\text {MarketBuyMax }(t)} \cdot x_{\text {MarketBuy }(t)} & \forall t \\
p_{\text {Buy }(t)} \geq P_{\text {MarketBuyMin }(t)} \cdot x_{\text {MarketBuy }(t)} & \forall t
\end{array}
$$

The market transactions in each period are unique:

$x_{\operatorname{MarketBuy}(t)}+x_{\text {MarketSell }(t)} \leq 1 \quad \forall t$

\section{7) Non-supplied demand constraint}

The NSD cannot be higher than the forecasted demand in scenario $s$ :

$$
p_{E N S(l, t, s)} \leq p_{\text {Load }(l, t, s)}-p_{\text {LoadDR(l,t,s) }} \quad \forall t, \forall l, \forall s
$$

\section{8) Generation curtailment power}

The generation curtailment power of non-dispatchable DG units cannot be higher than the predicted amount of generation:

$$
p_{G C P(i, t, s)} \leq P_{D G S c e n a r i o(i, t, s)} \quad \forall t, \forall i \in \Omega_{D G}^{n d}, \forall s
$$




\section{B. Benders' decomposition approach}

J. F. Benders [34] presented in 1962 a decomposition methodology to solve mixed integer problems. This method is based on the principle of the main problem decomposition into sub-problems. Benders decomposition method uses duality theory [35] in linear and nonlinear mathematical programming to divide a problem whose resolution is difficult in sub-problems. These sub-problems consider specific variables that are solved iteratively until the optimal solution is obtained [34]. The methodology considers all the variables involved and the concepts of external linearization, thus the dualization are applied.

Benders decomposition technique is adequate to solve large-scale problems like the ERM problem. The problem can be divided into a master problem and one or more slave problems. The master problem is usually classified as a linear or mixed integer problem including fewer technical constraints. The slave problems are linear or nonlinear and search if the solution of the master is technically feasible.

The master problem consists in finding the optimal solution without technical validation of the grid constraints, namely the DC-OPF (6). A simple balance equation is considered instead in the master, i.e. generation and load must match in each period. In the master problem, the binary variables are considered. The objective function of the master can be formulated as (32). In the case that any infeasibilities are found, one variable is added to the master problem, namely $\alpha$, which is designated by Benders' cuts.

Minimize $\quad E\left(O C_{\text {Total }}^{D+1}\right)+\alpha$

The Benders' cut is added in each iteration $m$ if any infeasibility is found in the slave problem and it is represented by (33)

$$
\begin{aligned}
& \alpha^{*} \geq Z_{u p}^{m-1}+ \\
& \sum_{t=1}^{T} \sum_{s p}^{N_{s p}} \lambda_{\text {Supplier }(s, t)}^{m-1} \cdot\left(x_{\text {Supplier }(s p, t)}^{m}-x_{\text {Supplier }(s p, t)}^{m-1}\right)+ \\
& \sum_{t=1}^{T} \sum_{i \in \Omega_{D G}^{d}} \lambda_{D G(i, t)}^{m-1} \cdot\left(x_{D G(i, t)}^{m}-x_{D G(i, t)}^{m-1}\right)+ \\
& \sum_{t=1}^{T} \sum_{e=1}^{N_{e}} \lambda_{E S S_{-} d s c(e, t, s)}^{m-1} \cdot\left(x_{E S S(e, t, s)}^{m}-x_{E S S(e, t, s)}^{m-1}\right)+ \\
& \sum_{t=1}^{T} \sum_{e=1}^{N_{e}} \lambda_{E S S_{-} c h g(e, t, s)}^{m-1} \cdot\left(y_{E S S(e, t, s)}^{m}-y_{E S S(e, t, s)}^{m-1}\right)+ \\
& \sum_{t=1}^{T} \sum_{v=1}^{N_{v}} \lambda_{E V_{-} d s c(v, t, s)}^{m-1} \cdot\left(x_{E V(v, t, s)}^{m}-x_{E V(v, t, s)}^{m-1}\right)+ \\
& \sum_{t=1}^{T} \sum_{v=1}^{N_{v}} \lambda_{E V_{-} \operatorname{chg}(v, t, s)}^{m-1} \cdot\left(y_{E V(v, t, s)}^{m}-y_{E V(v, t, s)}^{m-1}\right)
\end{aligned}
$$

The objective function of the slave problem is represented by (34) where the operation cost (1) and the relaxation variables $Z A$ and $Z F$ are minimized.
Minimize $E\left(O C_{\text {Total }}^{D+1}\right)+\sum_{t=1}^{T} \sum_{b=1}^{N_{b}} Z A_{(b, t)}+\sum_{t=1}^{T} \sum_{h=1}^{N_{h}} Z F_{(h, t)}$

In the slave problem (35) represents the power flow balance. The variable $Z A$ is added to allow feasible solutions. When $b=1$, the term $\left(p_{\text {Sell }(t)}-p_{B u y(t)}\right)$ is subtracted to the left part of the equation.

$$
\begin{aligned}
& \sum_{i \in \Omega_{D G}^{b}}\left(p_{D G(i, t)}-p_{G C P(i, t)}\right)+\sum_{S \in \Omega_{S}^{b}} p_{\text {Supplier }(s, t)}+ \\
& \sum_{l \in \Omega_{L}^{b}}\left(p_{N S D(l, t)}+p_{\text {Load } D R(l, t)}-p_{\text {Load }(l, t)}\right)+ \\
& \sum_{v \in \Omega_{V}^{b}}\left(p_{\text {Discharge }(v, t)}-p_{\text {Charge }(v, t)}\right)+ \\
& \sum_{e \in \Omega_{E}^{b}}\left(p_{\text {Discharge }(e, t)}-p_{\text {Charge }(e, t)}\right)- \\
& \sum_{h \in \Omega_{H}^{b}} p_{\text {Line }(h, t)}+Z A_{(b, t)}=0 \quad \forall t ; b \neq 1
\end{aligned}
$$

In, addition the lines power flow are relaxed by $Z F$ if congestion is verified as shown in (36) and (37):

$$
\begin{array}{ll}
p_{\text {Line }(h, t)}+Z F_{(h, t)} \leq P_{\text {LineMaxRate }(h)} & \forall t \\
p_{\text {Line }(h, t)}+Z F_{(h, t)} \geq-P_{\text {LineMaxRate }(h)} & \forall t
\end{array}
$$

\section{Evaluation metrics}

The advantage of the stochastic programming can be demonstrated by employing well-known developed indices in the literature, namely the expected value of perfect information (EVPI) and the value of stochastic solution (VSS) [35]. The EVPI represents the amount that the decision maker is not able to gain due to the presence of imperfect information, e.g. forecasts. It is useful to evaluate how the uncertainty factors affects the evaluated optimal problem. On the other hand, the VSS represents the advantage of using stochastic programming over a deterministic approach [35]. EVPI for minimization problems can be represented by (38). The stochastic solution, represented by $Z^{\mathrm{S}^{*}}$ is calculated by the stochastic programming approach and represents the total expected cost (1). $Z^{\mathrm{P} *}$, represents the wait-and-see solution (WSS). The WSS can be obtained by using the deterministic approach for each scenario. Then, WSS is computed by multiplying the individually obtained cost by each scenario probability.

$$
\mathrm{EVPI}=z^{\mathrm{S}^{*}}-z^{\mathrm{P} *}
$$

The VSS for minimization problems can be represented using (39). $Z^{\mathrm{D}^{*}}$ is the modified stochastic problem, which can be obtained by using a deterministic version with an average scenario. The results (only first-stage variables) are fixed in the original stochastic problem, thus obtaining $Z^{\mathrm{D}^{*}}$.

$$
\mathrm{VSS}=z^{\mathrm{D}^{*}}-z^{\mathrm{S}^{*}}
$$

\section{CASE STUdy}

In this section, a case study is presented to demonstrate how the proposed methodology is applied. A real distribution 
network from Portugal with 180 buses, $30 \mathrm{kV}$ and one substation [9] adapted to a future scenario is used in this paper. The network presents 90 load points, 5 parking lots for EVs, 116 DGs, one external supplier and 7 ESSs. The parking lots are distributed per 5 buses (3, 69, 96, 107 and 161). A subset of the original data was used from previous work [9], in which the EVs have been aggregated by bus, namely the aforementioned electrical buses. The external supplier is located at bus 1 corresponding to the substation location. DR with DLC contracts is considered in the case study. DLC cost considered is $0.02 \mathrm{~m} . \mathrm{u} . / \mathrm{kWh}$. The discharge prices are 0.18 m.u. $/ \mathrm{kWh}$ and $0.01 \mathrm{~m} . \mathrm{u} . / \mathrm{kWh}$ for EVs systems and ESS respectively. The ESS initial state of charge is considered zero, i.e., empty. The considered wholesale market energy price forecast is presented in Fig. 2 and took into account the Iberian market price for $10^{\text {th }}$ of January, 2017. Also, in this picture, it is presented the uncertainty of the market price forecast during the considered day. The prices and capacity of DG are based on the projections presented in [36]. Wind and solar power forecast as well as the demand forecast are presented in Fig. 3.

The scenarios are obtained using the methodology presented in Section III for 5000 scenarios and reduced to 150 scenarios, using GAMS/SCENRED. As can be seen, for instance, in Fig. 3 the wind and solar power generation forecast for period 12 varies between 2.59 MW and 3.01 MW, according to the scenario generation. The maximum standard deviation values for the considered uncertainty variables (demand, market, parking lots capacities, parking lots charge and discharge) are $15 \%, 10 \%, 35 \%, 35 \%$ and $35 \%$ respectively. The minimum values are respectively $8 \%, 6 \%$, $20 \%, 20 \%, 20 \%$.

As an example Fig. 4 depicts the box plot for the EVs battery capacity uncertainty in period 18 . Take as an example the parking lot 1 , located in bus 3 , it is possible to see a considerable uncertainty, varying between $0.05 \mathrm{MW}$ and a little more than $0.45 \mathrm{MW}$. Also, as can be seen, $50 \%$ of the values are located between $0.20 \mathrm{MW}$ and around $0.30 \mathrm{MW}$, corresponding to the interquartile range. $25 \%$ of values varying between $0.05 \mathrm{MW}$ and $0.20 \mathrm{MW}$ are located in the first quartile (Q1). Values between $0.05 \mathrm{MW}$ and $0.30 \mathrm{MW}$ (75\% of the values) are in the third quartile (Q3).

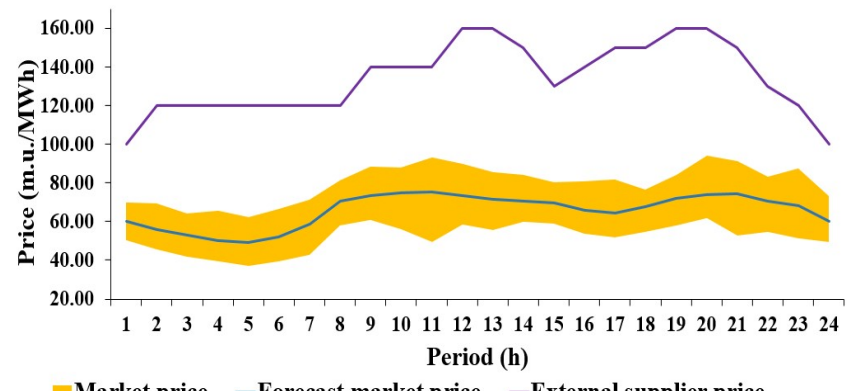

Market price - Forecast market price -External supplier price

Fig. 2. External supplier price and forecast of wholesale market price

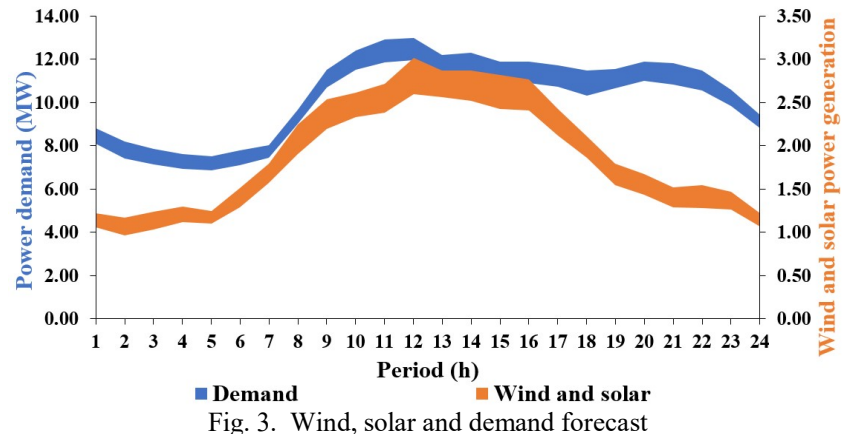

The energy resources data and prices are shown in Table I The market amount is set to $2 \mathrm{MW}$ in order to limit the exposure of the energy aggregator to higher uncertainty.

The case study presents four studies in order to show the impact of using storage and DR in the ERM concerning mitigation of uncertainty. The studies consider the following four cases: A - ESS and DR are considered; B - ESS and DR are not considered; $\mathrm{C}-\mathrm{ESS}$ is considered and DR is not; D DR is considered ESS is not.

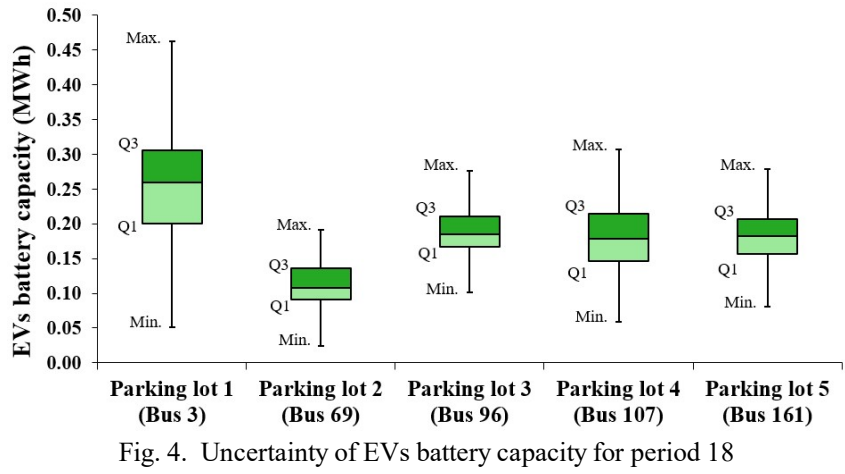

The proposed research work was developed in MATLAB R2014b and TOMLAB 8.164 bits (CPLEX version 12.5) using a computer with one Intel Xeon E5-2620 v2 processor and $16 \mathrm{~GB}$ of RAM running Windows 10 Pro.

TABLE I

CHARACTERIZATION OF 180-BUS DistribUTION NETWORK

\begin{tabular}{|c|c|c|c|c|c|}
\hline \multirow{2}{*}{\multicolumn{2}{|c|}{ Energy resources }} & $\begin{array}{c}\text { Prices } \\
\text { (m.u./MWh) }\end{array}$ & $\begin{array}{l}\text { Capacity } \\
\text { (MW) }\end{array}$ & $\begin{array}{l}\text { Forecast } \\
(\mathrm{MW})\end{array}$ & \multirow[t]{2}{*}{ Units } \\
\hline & & $\min -\max$ & $\min -\max$ & $\min -\max$ & \\
\hline \multicolumn{2}{|c|}{ Biomass } & $130-130$ & $0.02-6.23$ & & 17 \\
\hline \multicolumn{2}{|c|}{ Photovoltaic } & $150-150$ & & $0.00-0.36$ & 44 \\
\hline \multicolumn{2}{|c|}{ Wind } & $90-90$ & & $0.00-0.69$ & 55 \\
\hline \multicolumn{2}{|c|}{ External Supplier } & $100-160$ & $0.05-5.00$ & & 1 \\
\hline \multirow{2}{*}{ Storage } & Charge & $0-0$ & $0.00-1.20$ & & \multirow{2}{*}{7} \\
\hline & Discharge & $10-10$ & $0.00-1.20$ & & \\
\hline \multirow{2}{*}{$\begin{array}{l}\text { Parking } \\
\text { lots }\end{array}$} & Charge & $130-130$ & $0.31-1.01$ & & \multirow{2}{*}{5} \\
\hline & Discharge & $180-180$ & $0.31-1.00$ & & \\
\hline $\begin{array}{l}\text { Demand } \\
\text { Response }\end{array}$ & $\begin{array}{l}\text { Reduce } \\
\text { program }\end{array}$ & $20-20$ & $0.00-5.64$ & \multirow{3}{*}{$0.56-14.09$} & 90 \\
\hline \multicolumn{2}{|c|}{ Load } & $160-160$ & & & 90 \\
\hline \multicolumn{2}{|c|}{ Market buy and sell } & $45-84$ & $0.00-2.00$ & & 1 \\
\hline
\end{tabular}

\section{RESULTS AND DISCUSSION}

The proposed two-stage stochastic model is applied to the described case study in section IV, namely the four cases regarding DR and ESS availability. The optimization problem for the 150 scenarios deals with 1,239,721 variables, of which 
86,832 are integer variables and 215,188 constraints. The CPU time in each case has an average value of 2,700 seconds.

Fig. 5 and 6 present respectively the biomass and external supplier generation power for the four considered cases. Regarding biomass generation, the most considerable changes are in periods 1-2, 8-9 and 23-24. Regarding the external supplier, the changes are verified for the cases where the ESS is not considered (B and D). The change is a reduction of the generated power in some periods of the day, because the ESS is not charged in these cases.
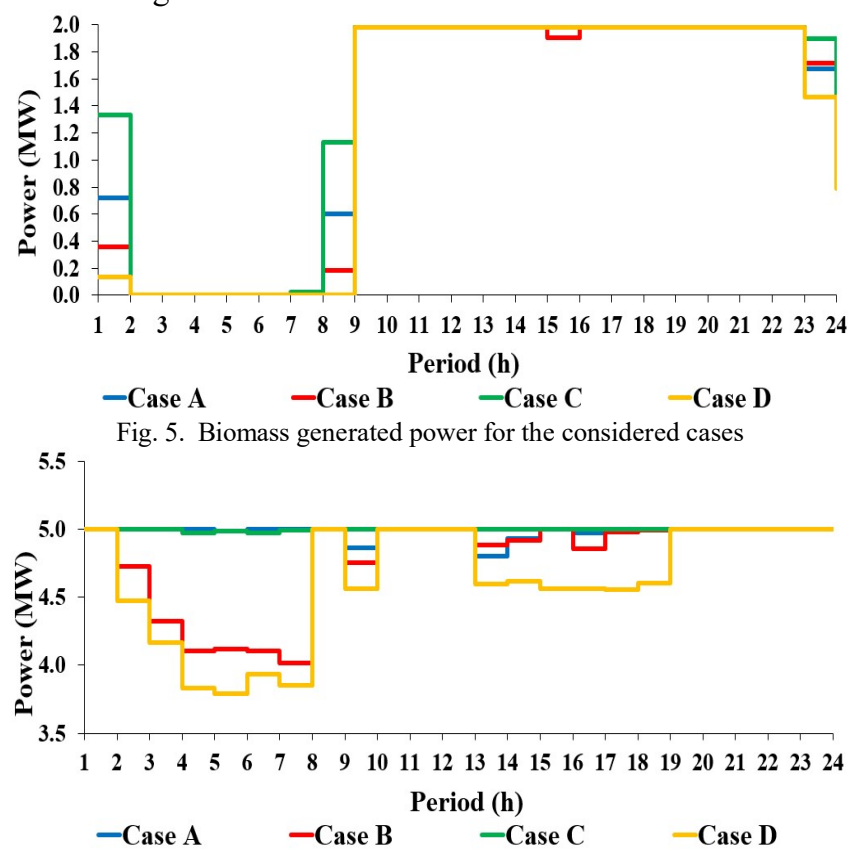

Fig. 6. External supplier generated power for the considered cases

The advantage to use the stochastic model (VSS) and the EVPI is shown in Fig. 7. Also, the cost for stochastic and deterministic models considering the four cases are presented in this figure. It is possible to see that the lower cost is verified when the ESS and DR are available. The case where both resources are unavailable presents the higher costs for both stochastic (47,208 m.u.) and deterministic (48,668 m.u.) models. For the cases $\mathrm{C}$ and $\mathrm{D}$, the costs for stochastic model are similar, but in the deterministic model the costs are $8.85 \%$ higher when the ESS is not available. Results also suggest that ESS contributes to avoid a higher cost when the deterministic model is used (case C). In case D, the DR resource is not so effective as ESS in case $\mathrm{C}$. The comparison between cases $\mathrm{C}$ and $\mathrm{D}$ is a good proof of the previous statement, where the VSS is higher in case D (11.75\%) which means that without ESS the stochastic model is more important to achieve lower expected costs mitigating the uncertainty.

Fig. 8 depicts the results of the stochastic scheduling of energy resources for cases $\mathrm{A}$ and $\mathrm{B}$, where it can be seen the minimum expected amount of energy for each resource, as well as the expected uncertainty. Regarding the wind and solar, the quantified uncertainty is $6.4 \mathrm{MWh}$ (case A and B).
This quantity represents the most probable variable amount. Concerning case A, the ESS and parking lots discharge and DR present an uncertainty of 11.29MWh, 1.24MWh and 7.81MWh respectively. The minimum expected values are $0.47 \mathrm{MWh}$ for ESS discharge and zero for the two other resources. For case B the uncertainty of parking lot discharge is $2.8 \mathrm{MWh}$ and the minimum expected value is zero.

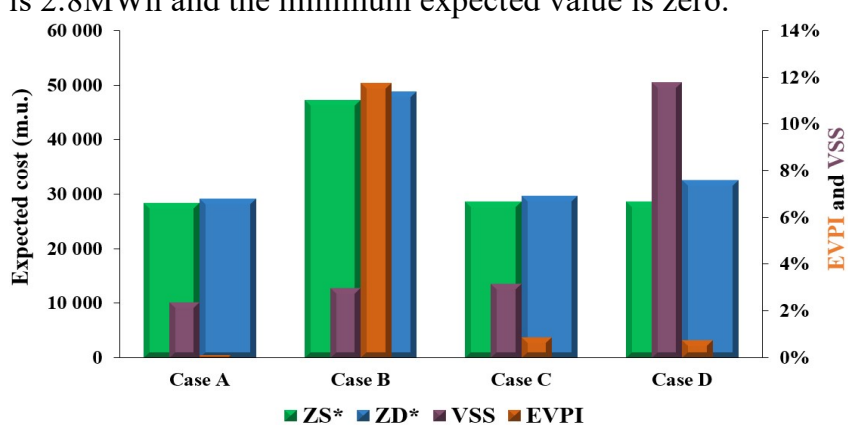

Fig. 7. EVPI and VSS for the considered cases

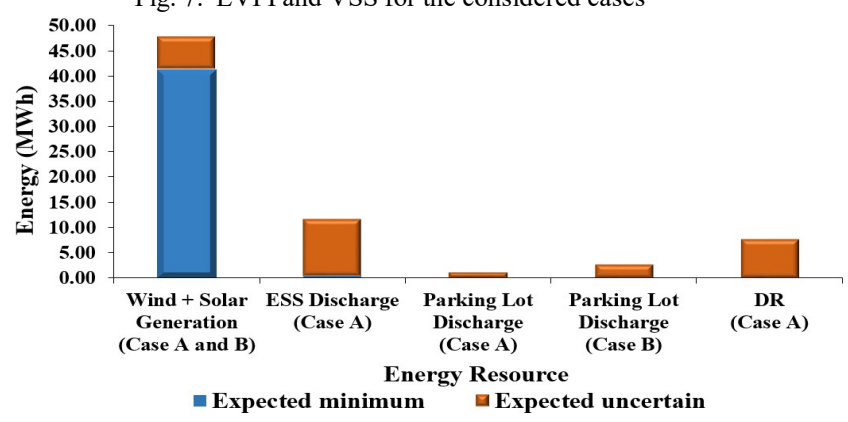

Fig. 8. Stochastic scheduling of energy resources for the case A and B)

In this case study, it was verified that the market results do not change. The market bid result is $2 \mathrm{MW}$ for every period, which corresponds to the maximum amount it can bid in the market (imposed in this case study).

\section{CONCLUSIONS}

A two-stage stochastic model using Benders' decomposition was proposed to solve the challenging problem of considering several sources of uncertainty in one integrated model and with network validation. The network constraints are validated for each scenario in the Benders' slave problem. The results indicate that the problem complexity can be reduced if the EVs are adequately aggregated (e.g. parking lot) instead of an individual control as in the previous work. Therefore, it is possible to increase the scalability of the model and consider several uncertainty sources. The research work also suggests that the increasing levels of uncertainty can be mitigated either with ESS or DR. In fact, the costs have been decreased by around $40 \%$ when ESS and DR have been both considered in the case study. In addition, in this particular case, the ESS enabled the reduction of the uncertainty impact more effectively than DR.

Future work should address how the nonlinearities can be tackled in the proposed stochastic model. New research may be based on hybrid versions of decomposition approaches, such as Dantzig-Wolfe or even evolutionary algorithms. 


\section{ACKNOWLEDGMENT}

This work has received funding from the European Union's Horizon 2020 research and innovation programme under the Marie Sklodowska-Curie grant agreement No 641794 (project DREAM-GO) and from FEDER Funds through COMPETE program and from National Funds through FCT under the project UID/EEA/00760/2013 and by NSF (US National Science Foundation) grant: IPP \#1312260. Bruno Canizes is supported by FCT Funds through the SFRH/BD/110678/2015 $\mathrm{PhD}$ scholarship. Mohammad Ali Fotouhi Ghazvini is supported by FCT Funds through the SFRH/BD/94688/2013 $\mathrm{PhD}$ scholarship.

\section{REFERENCES}

[1] R. Ambrosio, "Transactive Energy Systems," IEEE Electrif. Mag., vol. 4, no. 4, pp. 4-7, Dec. 2016

[2] F. Rahimi and A. Ipakchi, "Using a Transactive Energy Framework Providing Grid Services from Smart Buildings," IEEE Electrif. Mag., vol. 4, no. 4, pp. 23-29, Dec. 2016.

[3] Shi You, C. Traeholt, and B. Poulsen, "A market-based Virtual Power Plant," in 2009 International Conference on Clean Electrical Power, 2009, pp. 460-465.

[4] A. G. Zamani, A. Zakariazadeh, S. Jadid, and A. Kazemi, "Stochastic operational scheduling of distributed energy resources in a large scale virtual power plant," Int. J. Electr. Power Energy Syst., vol. 82, pp. 608-620, 2016

[5] R. Hemmati, H. Saboori, and S. Saboori, "Assessing wind uncertainty impact on short term operation scheduling of coordinated energy storage systems and thermal units," Renew. Energy, vol. 95, pp. 74-84, 2016

[6] D. T. Ton and W.-T. P. Wang, "A more resilient grid: The U.S. Department of Energy joins with stakeholders in an R\&D plan," IEEE Power Energy Mag., vol. 13, no. 3, pp. 26-34, 2015.

[7] J. Soares, T. Sousa, H. Morais, Z. Vale, B. Canizes, and A. Silva, "Application-Specific Modified Particle Swarm Optimization for energy resource scheduling considering vehicle-to-grid," Appl. Soft Comput., vol. 13, no. 11, pp. 4264-4280, 2013.

[8] A. Zakariazadeh, S. Jadid, and P. Siano, "Multi-objective scheduling of electric vehicles in smart distribution system," Energy Convers. Manag., vol. 79, pp. 43-53, Mar. 2014.

[9] J. Soares, B. Canizes, Z. Vale, and G. K. Venayagamoorthy, "Benders' Decomposition Applied to Energy Resource Management in Smart Distribution Networks," in Clemson University Power System Conference (PSC) 2016, 2016.

[10]A. Chaouachi, R. M. Kamel, R. Andoulsi, and K. Nagasaka, "Multiobjective Intelligent Energy Management for a Microgrid," IEEE Trans. Ind. Electron., vol. 60, no. 4, pp. 1688-1699, Apr. 2013.

[11]C. Chen, S. Duan, T. Cai, B. Liu, and G. Hu, "Smart energy management system for optimal microgrid economic operation," IET Renew. Power Gener., vol. 5, no. 3, p. 258, 2011

[12]J. Wang, C. Liu, D. Ton, Y. Zhou, J. Kim, and A. Vyas, "Impact of plug-in hybrid electric vehicles on power systems with demand response and wind power," Energy Policy, vol. 39, no. 7, pp. 4016-4021, 2011.

[13]J. Soares, H. Morais, T. Sousa, Z. Vale, and P. Faria, "Day-Ahead Resource Scheduling Including Demand Response for Electric Vehicles," Smart Grid, IEEE Trans., vol. 4, no. 1, pp. 596-605, 2013.

[14]J. Soares, M. A. Fotouhi Ghazvini, M. Silva, and Z. Vale, "Multidimensional signaling method for population-based metaheuristics: Solving the large-scale scheduling problem in smart grids," Swarm Evol. Comput., vol. 29, pp. 13-32, 2016.

[15]J. Soares, M. A. Fotouhi Ghazvini, Z. Vale, and P. B. de Moura Oliveira, "A multi-objective model for the day-ahead energy resource scheduling of a smart grid with high penetration of sensitive loads," Appl. Energy, vol. 162, pp. 1074-1088, Jan. 2016.

[16]M. Liu, F. L. Quilumba, and W. J. Lee, "Dispatch Scheduling for a Wind Farm With Hybrid Energy Storage Based on Wind and LMP Forecasting," IEEE Transactions on Industry Applications, vol. 51, no. 3. pp. 1970-1977, 2015.

[17]A. N. Ghalelou, A. P. Fakhri, S. Nojavan, M. Majidi, and H. Hatami, "A stochastic self-scheduling program for compressed air energy storage (CAES) of renewable energy sources (RESs) based on a demand response mechanism," Energy Convers. Manag., vol. 120, pp. 388-396, 2016.

[18]M. Rahimiyan and L. Baringo, "Strategic Bidding for a Virtual Power Plant in the Day-Ahead and Real-Time Markets: A Price-Taker Robust Optimization Approach," IEEE Trans. Power Syst., vol. 31, no. 4, pp. 2676-2687, Jul. 2016.

[19]L. Baringo and A. J. Conejo, "Offering Strategy of Wind-Power Producer: A Multi-Stage Risk-Constrained Approach," IEEE Trans. Power Syst., vol. 31, no. 2, pp. 1420-1429, 2016.

[20]A. A. Eajal, M. F. Shaaban, K. Ponnambalam, and E. F. El-Saadany, "Stochastic Centralized Dispatch Scheme for AC/DC Hybrid Smart Distribution Systems," IEEE Trans. Sustain. Energy, pp. 1-14, 2016.

[21]Z. Ding and W. J. Lee, "A Stochastic Microgrid Operation Scheme to Balance Between System Reliability and Greenhouse Gas Emission," IEEE Transactions on Industry Applications, vol. 52, no. 2. pp. 1157 1166,2016

[22]A. G. Zamani, A. Zakariazadeh, and S. Jadid, "Day-ahead resource scheduling of a renewable energy based virtual power plant," Appl. Energy, vol. 169, pp. 324-340, May 2016.

[23]L. Ju, Z. Tan, J. Yuan, Q. Tan, H. Li, and F. Dong, "A bi-level stochastic scheduling optimization model for a virtual power plant connected to a wind-photovoltaic-energy storage system considering the uncertainty and demand response," Appl. Energy, vol. 171, pp. 184199, 2016.

[24]J. Soares, M. A. F. Ghazvini, N. Borges, and Z. Vale, "A Stochastic Model for Energy Resources Management in Smart Grids," Electr. Power Syst. Res., 2016.

[25]H. Wu and M. Shahidehpour, "A Game Theoretic Approach to RiskBased Optimal Bidding Strategies for Electric Vehicle Aggregators in Electricity Markets With Variable Wind Energy Resources," vol. 7, no. 1, pp. 374-385, 2016.

[26]M. A. Fotouhi Ghazvini, P. Faria, S. Ramos, H. Morais, and Z. Vale, "Incentive-based demand response programs designed by asset-light retail electricity providers for the day-ahead market," Energy, vol. 82, pp. 786-799, 2015.

[27]H. Wu, M. Shahidehpour, A. Alabdulwahab, and A. Abusorrah, "Thermal Generation Flexibility with Ramping Costs and Hourly Demand Response in Stochastic Security-Constrained Scheduling of Variable Energy Sources," IEEE Trans. Power Syst., vol. 30, no. 6, pp. 2955-2964, 2015.

[28]E. A. Bakirtzis, A. V. Ntomaris, E. G. Kardakos, C. K. Simoglou, P. N. Biskas, and A. G. Bakirtzis, "A unified unit commitment - Economic dispatch model for short-term power system scheduling under high wind energy penetration," Int. Conf. Eur. Energy Mark. EEM, 2014.

[29]A. Nasri, S. J. Kazempour, A. J. Conejo, and M. Ghandhari, "Networkconstrained AC unit commitment under uncertainty: A benders' decomposition approach," IEEE Trans. Power Syst., vol. 31, no. 1, pp. $412-422,2016$

[30]N. Gröwe-Kuska, H. Heitsch, and W. Römisch, "Scenario reduction and scenario tree construction for power management problems," in 2003 IEEE Bologna PowerTech - Conference Proceedings, 2003, vol. 3, pp. $152-158$.

[31]I. Momber, A. Siddiqui, T. G. S. Roman, and L. Soder, "Risk Averse Scheduling by a PEV Aggregator Under Uncertainty," IEEE Trans. Power Syst., vol. 30, no. 2, pp. 882-891, 2014.

[32]J. R. Birge and F. Louveaux, Introduction to stochastic programming. Springer Science \& Business Media, 2011.

[33]C. Tomlinson, J. Cainey, and A. Price, "Local energy markets," LEMMA project. Swanbarton \& Information Processing Limited, United Kingdom, 2013.

[34]A. J. Conejo, E. Castillo, R. Minguez, and R. Garcia-Bertrand, Decomposition Techniques in Mathematical Programming: Engineering and Science Applications. Springer, 2006.

[35]A. J. Conejo, M. Carrión, and J. M. Morales, Decision Making Under Uncertainty in Electricity Markets, vol. 153. Boston, MA: Springer US, 2010.

[36]J. M. Christine Lins, Arthouros Zervos, "Re-thinking 2050: A 100\% Renewable Energy Vision for the European Union,” 2010. 